\title{
Symmetry dependence and universality of practical algebraic functionals in density-matrix-functional theory
}

\author{
Oleg V. Gritsenko, ${ }^{1,2,4}$ Jian Wang, ${ }^{1, *}$ and Peter J. Knowles ${ }^{3}$ \\ ${ }^{1}$ School of Science, Huzhou University, Zhejiang 10083, China \\ ${ }^{2}$ Section Theoretical Chemistry, Vrije Universiteit, De Boelelaan 1083, 1081 HV, Amsterdam, The Netherlands \\ ${ }^{3}$ School of Chemistry, Cardiff University, Main Building, Park Place, Cardiff CF10 3AT, United Kingdom \\ ${ }^{4}$ Institute of Physics, Lodz University of Technology, ul. Wolczanska 219, 90-924 Lodz, Poland
}

(Received 30 January 2019; published 24 April 2019; corrected 30 April 2019)

\begin{abstract}
When dealing with a fully symmetrical ground state, the symmetry dependence of the universal HohenbergKohn energy functional $F[\gamma]$ of the first-order reduced density matrix (RDM) $\gamma$ can be conveniently neglected. The situation changes drastically in the case of the dissociation of a symmetrical molecule with the state crossing, in the course of which the potential energy curve of the initial non-fully symmetrical ground state is eventually crossed with that of the fully symmetrical state. In this case, as is demonstrated in the present paper, the second-order RDM $\Gamma_{i j, k l}$ in the representation of the natural orbitals (NOs) is symmetry dependent. Since $\Gamma_{i j, k l}$ is the goal in the design of $\Gamma_{i j, k l}(n)$ as a functional of NO occupations $\{n\}$, which is part of a practical density matrix functional $F[\gamma], \Gamma_{i j, k l}(n)$ must also depend on the symmetry, especially the irreducible representation of the symmetry group. The result has immediate implications for study of structural (or phase) transitions based on a single symmetry-independent functional. The demonstration is given in the minimal-base model of the dissociation of the prototype $\mathrm{H}_{4}$ molecule in the rhombic structure.
\end{abstract}

DOI: 10.1103/PhysRevA.99.042516

\section{INTRODUCTION}

The key quantity of both density (DFT) and density matrix (DMFT) functional theories is the sum $F$ of the kinetic $T$ and electron-electron interaction $V_{e e}$ energies. According to the second Hohenberg-Kohn (HK) theorem [1] and its DMFT extension by Gilbert [2], for the lowest state $\Psi, F[\mu]$ is the universal functional of the electron density $\mu=\rho$,

$$
\rho(\boldsymbol{x})=\sum_{i} n_{i}\left|\chi_{i}(\boldsymbol{x})\right|^{2},
$$

in DFT or of the first-order reduced density matrix (1RDM) $\mu=\gamma$,

$$
\gamma\left(\boldsymbol{x}, \boldsymbol{x}^{\prime}\right)=\sum_{i} n_{i} \chi_{i}^{*}(\boldsymbol{x}) \chi_{i}\left(\boldsymbol{x}^{\prime}\right),
$$

in DMFT. Here, $\rho$ and $\gamma$ are expressed through the canonical orbitals of the theories, which are the Kohn-Sham (KS) orbitals $\phi_{i}(\boldsymbol{x})$ and the natural orbitals (NOs) $\chi_{i}(\boldsymbol{x})(\boldsymbol{x}$ stands for both spatial $\boldsymbol{r}$ and the spin $s$ coordinates) as well as through their occupation numbers (ONs) $n_{i}$. The former orbitals have the integer ONs while the latter have fractional ONs.

In DFT $F[\rho]$ is subdivided to the noninteracting kinetic energy $T_{s}[\{\phi\}]$ and the Hartree-exchange-correlation (Hxc) energy $E_{H x c}[\rho]$ functionals

$$
\begin{aligned}
F[\rho] & =T_{s}[\{\phi\}]+E_{H x c}[\rho] \\
& =-\frac{1}{2} \sum_{i} n_{i}\left\langle\phi_{i}\left|\nabla^{2}\right| \phi_{i}\right\rangle+E_{H x c}[\rho],
\end{aligned}
$$

\footnotetext{
*jwang572@hotmail.com
}

so $E_{H x c}[\rho]$ requires the interacting correction to the kinetic contribution. The exact form of the DFT functional is still unknown, but it was claimed that the functional is universal in the sense that it does not depend on the external potential [1]. The universal property of $F[\rho]$ in DFT has since been taken for granted, even for an approximate functional. Once a new functional is developed for a set of specific systems, it will be automatically applied to other systems. However, most DFT functionals become less reliable beyond systems on which the model functional was trained [3].

In DMFT, the kinetic energy $T$ is expressed exactly with the natural orbitals,

$$
T[\{\chi\},\{n\}]=-\frac{1}{2} \sum_{i} n_{i}\left\langle\chi_{i}\left|\nabla^{2}\right| \chi_{i}\right\rangle,
$$

so only the electron-electron potential energy need be modelled as a functional:

$$
F[\gamma]=-\frac{1}{2} \sum_{i} n_{i}\left\langle\chi_{i}\left|\nabla^{2}\right| \chi_{i}\right\rangle+V_{e e}[\{\chi\},\{n\}] .
$$

Even though the exact form of the functional $V_{e e}[\{\chi\},\{n\}]$ is still unknown, its value can be calculated alternatively as the wave function $\Psi$ is known:

$$
\left\langle\Psi\left|V_{e e}\right| \Psi\right\rangle=\frac{1}{2} \sum_{i j k l}\left\langle\Psi\left|a_{i}^{\dagger} a_{j}^{\dagger} a_{l} a_{k}\right| \Psi\right\rangle\langle i j \mid k l\rangle,
$$

where $\left\langle\Psi\left|a_{i}^{\dagger} a_{j}^{\dagger} a_{l} a_{k}\right| \Psi\right\rangle$ is the second-order reduced density matrix (2RDM), $a_{i}^{\dagger}$ and $a_{k}$ are the creation and annihilation operators, and $\langle i j \mid k l\rangle$ is the two-electron integral in 
physicists' notation:

$$
\langle i j \mid k l\rangle=\int \frac{\chi_{i}^{*}\left(\boldsymbol{x}_{1}\right) \chi_{j}^{*}\left(\boldsymbol{x}_{2}\right) \chi_{k}\left(\boldsymbol{x}_{1}\right) \chi_{l}\left(\boldsymbol{x}_{2}\right)}{\left|\boldsymbol{r}_{1}-\boldsymbol{r}_{2}\right|} d \boldsymbol{x}_{1} d \boldsymbol{x}_{2} .
$$

The key to model the functional in DMFT is to replace the 2RDM with a functional of ONs and NOs. This is, however, a nontrivial task. Since the orbitals are already contained in the two-electron integral, the conventional wisdom is to assume that the remaining content of the functional is a function of ONs only, i.e.,

$$
\left\langle\Psi\left|a_{i}^{\dagger} a_{j}^{\dagger} a_{l} a_{k}\right| \Psi\right\rangle=\Gamma_{i j, k l}(n) .
$$

Almost all model DMFT functionals in practice [4-11] are thus in the form of $\Gamma_{i j, k l}(n)$. In some special cases, such a functional form of $\Gamma_{i j, k l}(n)$ can even be exact-for example, the two-electron systems [12] and the translationally invariant one-band lattice [13].

In Ref. [14], nonuniqueness of the function $\Gamma_{i j, k l}(n)$ was established by demonstrating that systems with the same ON spectrum can have different 2RDMs. This finding indicates that, besides the ONs, $\Gamma_{i j, k l}$ depends on the additional parameters $\{P\}$. Based on Levy's argument [15], one of the current authors [16] proposed the total symmetry $S(I)$ of the generic wave function $\Psi$, where $S$ is the symmetry group of the system and $I$ is its particular irreducible representation (irrep), as possible additional parameters. While the argument is correct in principle, numerical evidences still fall short of ruling out the possibility of nonuniqueness for the functional form $\Gamma_{i j, k l}(n)$ [17]. The possible symmetry dependence of $\Gamma_{i j, k l}(n)$, however, raises a question regarding its universality. In 1976, Gunnarsson and Lundqvist [18] extended the KohnSham scheme to the lowest excited state with a specified symmetry of total spin angular momenta. They noted that unlike the ground-state functional, the functional will be no longer universal but depends on the quantum numbers due to the spin symmetry. The topic was also considered by von Barth [19]. While these earlier works concerned on the spin symmetry and the excited state, here we focus on the spatial symmetry and the ground state, which is the main target of DFT and DMFT applications.

\section{SYMMETRY DEPENDENCE OF ALGEBRAIC FUNCTIONAL $\boldsymbol{\Gamma}_{i j, k l}(n)$ IN MODEL SYSTEMS}

We will elaborate on the paradigmatic four-electron $\mathrm{H}_{4}$ molecule. The geometry and symmetry of the $\mathrm{H}_{4}$ molecule can be easily modified to have a square, a rectangle, a rhombus, etc. Extensive configuration interaction (CI) calculations for this system have already been reported [20,21]. Of particular interest to us is the ground state of rhombic $\mathrm{H}_{4}$, which belongs to the non-fully symmetrical $B_{1 g}$ irreducible representation of the $D_{2 h}$ group. In the course of the partial dissociation to a $\mathrm{H}_{2}+2 \mathrm{H}$ molecule $\left(\mathrm{a}_{2}\right.$ molecule plus two separated $\mathrm{H}$ atoms at far distance),

$$
\mathrm{H}_{4} \rightarrow \mathrm{H}_{2}+2 \mathrm{H},
$$

its symmetry changes to the $A_{g}$ irreducible representation. The above-mentioned features make this system ideal to check the supposed symmetry dependence of $\Gamma_{i j, k l}(n)$.
For our analysis, we adopt the minimal four-orbital model for the $\mathrm{H}_{4}$ molecule as previous CI calculations [20,21]. The $1 \mathrm{RDM}$ of the model has the following form in terms of spinNOs:

$$
\gamma_{v}\left(\boldsymbol{x}, \boldsymbol{x}^{\prime}\right)=\sum_{\sigma=\alpha, \beta} \sum_{p=1}^{4} n_{p \sigma} \chi_{p \sigma}^{*}(\boldsymbol{x}) \chi_{p \sigma}\left(\boldsymbol{x}^{\prime}\right) .
$$

With the four $\mathrm{H}$ atoms located on the axes, the $\mathrm{CI}$ wave function of the rhombic $\mathrm{H}_{4}$ structure belonging to the $B_{1 g}$ irrep of the $D_{2 h}$ symmetry group has the following form:

$$
\begin{aligned}
\Psi= & c_{1}\left|\left(\chi_{1}^{a_{1 g}}\right)^{2} \chi_{2}^{e_{u 1}} \chi_{3}^{e_{u 2}}(\alpha \beta-\beta \alpha)\right| \\
& +c_{2}\left|\left(\chi_{4}^{b_{2 g}}\right)^{2} \chi_{2}^{e_{u 1}} \chi_{3}^{e_{u 2}}(\alpha \beta-\beta \alpha)\right| \\
& +c_{3} \mid \chi_{1}^{a_{1 g}} \chi_{2}^{e_{u 1}} \chi_{3}^{e_{u 2}} \chi_{4}^{b_{2 g}}[\alpha \alpha \beta \beta+\beta \beta \alpha \alpha \\
& +\alpha \beta \alpha \beta+\beta \alpha \beta \alpha-2(\alpha \beta \beta \alpha+\beta \alpha \alpha \beta)],
\end{aligned}
$$

where the orbital superscripts indicate the symmetry of the NOs $\chi_{p}$.

The ONs $n_{p \sigma}$ of the corresponding spin NOs $\chi_{p \sigma}$ are as follows:

$$
\begin{gathered}
n_{1 \sigma}=2 c_{1}^{2}+6 c_{3}^{2}, \\
n_{4 \sigma}=1-n_{1 \sigma},
\end{gathered}
$$

and

$$
n_{2 \sigma}=n_{3 \sigma}=c_{1}^{2}+c_{2}^{2}+6 c_{3}^{2}=\frac{1}{2} .
$$

That is because the wave function is normalized as $2 c_{1}^{2}+$ $2 c_{2}^{2}+12 c_{3}^{2}=1$.

The geometry of $\mathrm{H}_{2}+2 \mathrm{H}$ can be formed by moving one pair of opposite $\mathrm{H}$ atoms of the rhombus to infinity. Interestingly, the irreducible representation of $\mathrm{H}_{2}+2 \mathrm{H}$ is no longer $B_{1 g}$ but becomes $A_{g}$ of the $D_{2 h}$ symmetry group. The wave function is

$$
\Psi=\left|\left[c_{g}\left(\chi_{1}^{\sigma_{g}}\right)^{2}+c_{u}\left(\chi_{4}^{\sigma_{u}}\right)^{2}\right] \chi_{2}^{1 s_{A}} \chi_{3}^{1 s_{B}} \sqrt{\frac{1}{2}}(\alpha \beta-\beta \alpha)\right|,
$$

with $c_{u}=-\sqrt{1-c_{g}^{2}}$. Here, $\chi_{1}^{\sigma_{g}}$ and $\chi_{4}^{\sigma_{u}}$ are the bonding and antibonding NOs of the $\mathrm{H}_{2}$ fragment, while $\chi_{2}^{1 s_{A}}$ and $\chi_{3}^{1 s_{B}}$ are the orbitals of the remote individual $\mathrm{H}$ atoms $A$ and $B$. The corresponding ONs are

$$
\begin{gathered}
n_{1 \sigma}=c_{g}^{2}, \\
n_{4 \sigma}=1-n_{1 \sigma},
\end{gathered}
$$

and

$$
n_{2 \sigma}=n_{3 \sigma}=\frac{1}{2}
$$

In order to establish that the considered wave functions (11), on the one hand, and (15), on the other hand, produce different functionals $\Gamma_{i j, k l}(n)$, one has to construct and to compare the 2RDMs for the same ONs. Following the previous paper [14], we choose the compact $\mathrm{H}_{4}$ square structure with the $\mathrm{H}$-H side length $R_{4}=2.0 \AA$. This yields $n_{1 \sigma}=0.779007$ and $n_{4 \sigma}=0.220993$. The same ONs are produced for the 
rhombic $\mathrm{H}_{2}+2 \mathrm{H}$ structure with a $\mathrm{H}-\mathrm{H}$ bond length $R_{2}=$ $1.79326407 \AA$.

For the rhombic $\mathrm{H}_{4}$, one can either vary the $\mathrm{H}-\mathrm{H}$ bond length at a given apex again, or vary the apex angle at a given bond length. For example, when we fix one apex angle at $100^{\circ}$, we find the same ON spectrum of $n_{1 \sigma}=0.779007, n_{2 \sigma}=$ $n_{3 \sigma}=0.5$, and $n_{4 \sigma}=0.220993$ when $R=1.997380 \AA$. If the apex angle is $120^{\circ}$, the bond length should be $R=$ $1.9726658 \AA$ to produce the same ON spectrum.
Now the chosen structures here, the square $\mathrm{H}_{4}$, the rhombic $\mathrm{H}_{4}$, and then the rhombic $\mathrm{H}_{2}+2 \mathrm{H}$, all have the same ONs. If the functional $\Gamma_{i j, k l}(n)$ depends only on the ONs and if it is universal, it should be the same for all four structures. So in the following, we calculate their $2 \mathrm{RDM}$ s to check if this is the case.

The 2RDMs are defined as $\Gamma_{i j, k l}=\left\langle\Psi\left|a_{i}^{\dagger} a_{j}^{\dagger} a_{l} a_{k}\right| \Psi\right\rangle$. For the $\mathrm{H}_{2}+2 \mathrm{H}$ structure the nonredundant same-spin block of $\Gamma_{i j, k l}$ with $i<j, k<l$ has the following form:

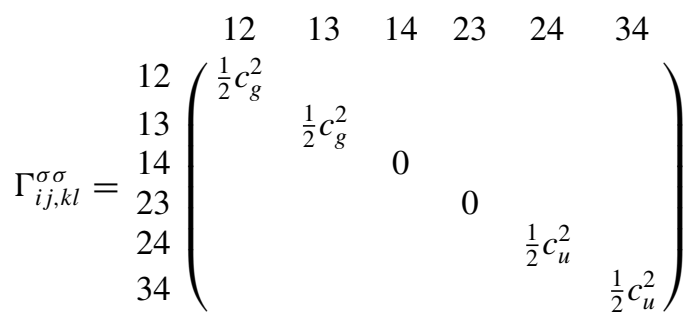

while the opposite-spin block is

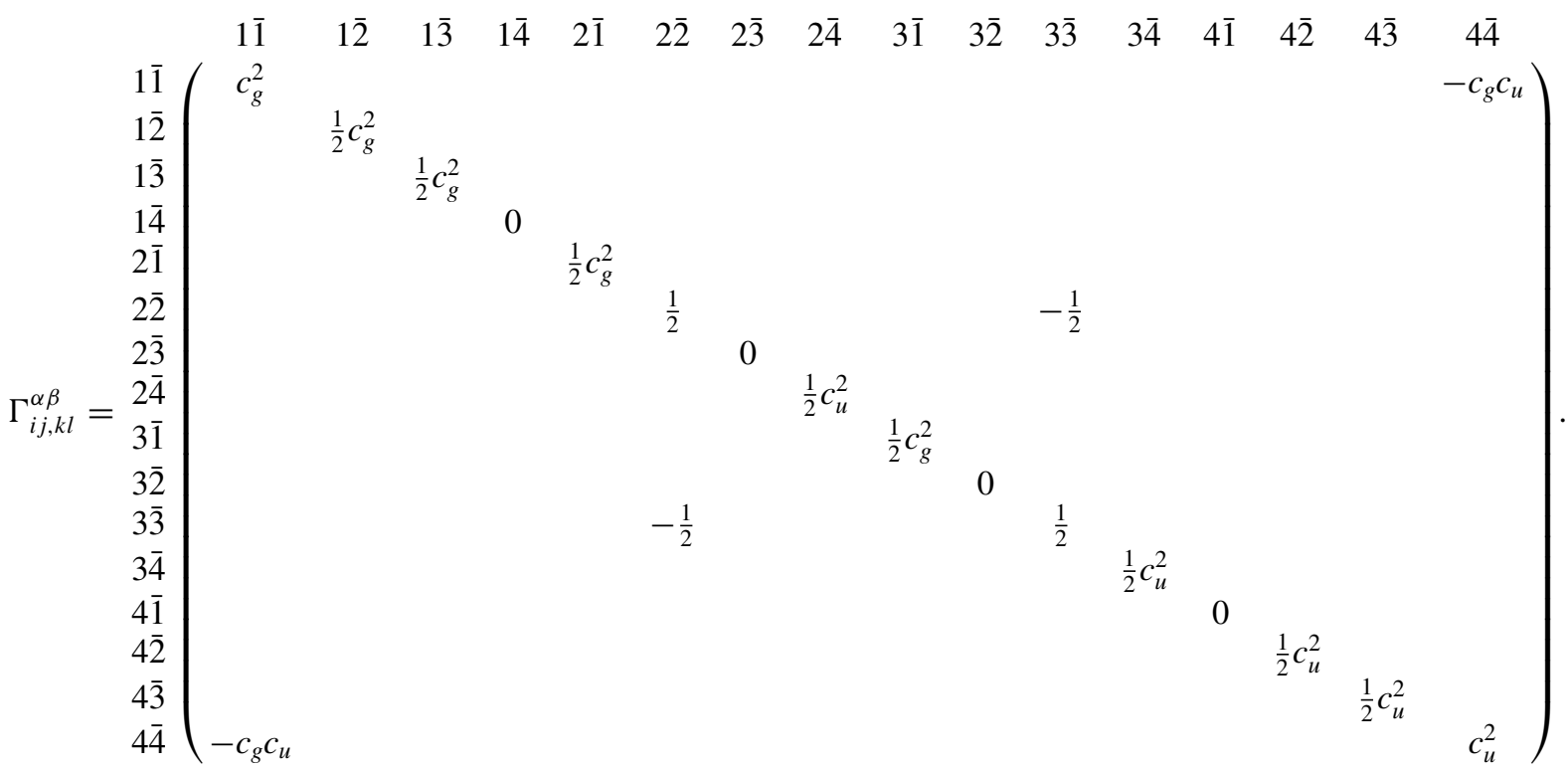

The labeling above and on the left of the matrix shows the indices $i j, k l$, with the orbitals ordered as $\sigma_{g}, A, B, \sigma_{u}\left(\mathrm{H}_{2}+2 \mathrm{H}\right)$. Here, the indices with bars indicate $\beta$-spin orbitals. For the rhombic $\mathrm{H}_{4}$ structure, using the wave function above, we obtain the nonredundant same-spin block $\Gamma_{i j, k l}^{\sigma \sigma}$, with $i<j, k<l$ as

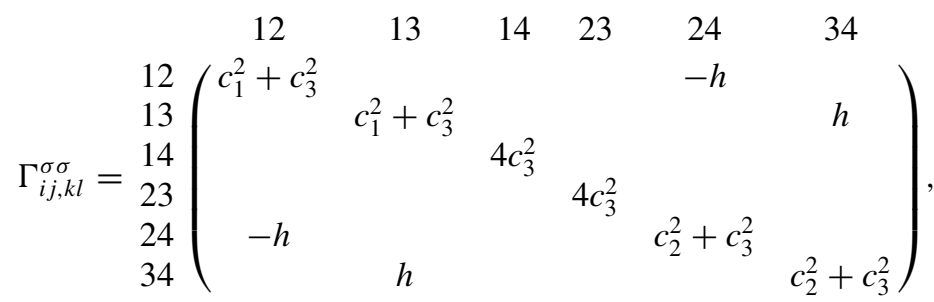

where $h=c_{3}\left(c_{2}-c_{1}\right)$, with the orbitals ordered as $a_{1 g}, e_{u 1}, e_{u 2}, b_{2 g}$. 
The opposite-spin block $\Gamma_{i j, k l}^{\sigma \beta}$ is

$1 \overline{1}$
$1 \overline{2}$
$1 \overline{3}$
$1 \overline{1}$

where $a=c_{1}^{2}+5 c_{3}^{2}, b=c_{2}^{2}+5 c_{3}^{2}, d=c_{1}^{2}+c_{2}^{2}, e=c_{3}^{2}, h=$ $c_{3}\left(c_{2}-c_{1}\right)$. The notations here are similar to those used in the previous paper [14].

Table I compares the eigenvalues of $\Gamma_{i j, k l}$ normalized to $N(N-1) / 2=6$ for the three structures. The square $\mathrm{H}_{4}$ can be considered a special case of the rhombus, while the apex angle becomes $90^{\circ}$. Its irreducible representation is ${ }^{1} B_{1 g}$ in the $D_{4 h}$ group, which can be correlated to the $B_{1 g}$ in the $D_{2 h}$ group of the rhombus.

One can see from Table I that variation of the geometry of $\mathrm{H}_{4}$ (which implies change of the external potential), when going from the square to the rhombi with apex angles $100^{\circ}$ and $120^{\circ}$, does not produce a noticeable change of the 2RDM eigenvalues. This conveys the universality property of the functional $\Gamma_{i j, k l}(n)$ via Eq. (8). On the other hand, the irreducible representation change from $B_{1 g}$ to $A_{g}$ when going from $\mathrm{H}_{4}$ to $\mathrm{H}_{2}+2 \mathrm{H}$ does produce a profound change of the 2RDMs [see Table I and compare Eq. (19) with Eq. (21), and

TABLE I. Eigenvalues of the 2RDM for the rhombic $\mathrm{H}_{2}+2 \mathrm{H}$, the square $\mathrm{H}_{4}$, and the rhombus $\mathrm{H}_{4}$ with the apex angle $100^{\circ}$ and $120^{\circ}$, respectively. The specifications for geometry are $R_{2}=$ $1.79326407 \AA$ for the rhombic $\mathrm{H}_{2}+2 \mathrm{H}, R_{4}=2.0 \AA$ for the square $\mathrm{H}_{4}, R=1.997380 \AA$ for the rhombus with an apex angle of $100^{\circ}$, and $R=1.9726658 \AA$ with an apex angle of $120^{\circ}$. The numbers in brackets are the degeneracies.

\begin{tabular}{lccc}
\hline \hline $\mathrm{H}_{2}+2 \mathrm{H}$ & Square & Rhombus $\left(100^{\circ}\right)$ & Rhombus $\left(120^{\circ}\right)$ \\
\hline $1.0000000(2)$ & $0.8033100(2)$ & $0.8033851(2)$ & $0.8040501(2)$ \\
$0.3895033(8)$ & $0.6966765(2)$ & $0.6966021(2)$ & $0.6959426(2)$ \\
$0.1104966(8)$ & $0.3926620(6)$ & $0.3926665(6)$ & $0.3927050(6)$ \\
$0.0000000(10)$ & $0.0655633(6)$ & $0.0655382(6)$ & $0.0653166(6)$ \\
& $0.0417746(6)$ & $0.0417951(6)$ & $0.0419783(6)$ \\
& $0.0000133(2)$ & $0.0000126(2)$ & $0.0000072(2)$ \\
& $0.0000000(4)$ & $0.0000000(4)$ & $0.0000000(4)$ \\
\hline \hline
\end{tabular}

Eq. (20) with Eq. (22)]. The results suggest a single functional $\Gamma_{i j, k l}^{B_{1 g}}(n)$ for all $\mathrm{H}_{4}$ structures and an entirely different functional $\Gamma_{i j, k l}^{A_{g}}(n)$ for $\mathrm{H}_{2}+2 \mathrm{H}$.

A possible reason for the established nonuniqueness [14] of $\Gamma_{i j, k l}(n)$ might be just a failure of the too-restrictive dependence of $\Gamma_{i j, k l}$ on only ONs, which is conventionally assumed in DMFT [4-11]. Such a failure can easily lead to a nondesirable dependence of $\Gamma_{i j, k l}$ on the external potential (or geometry), which would compromise the important universality of the functional according to the HK theorem. On the other hand, the present results suggest an alternative explanation that the functional form of $\Gamma_{i j, k l}(n)$ should be symmetry dependent. Specifically, in the considered example, the 2RDM function becomes a piecewise sequence of symmetry-dependent fragments $\Gamma_{i j, k l}^{B_{1 g}}(n)$ and $\Gamma_{i j, k l}^{A_{g}}(n)$. Since $\Gamma_{i j, k l}(n)$ is a part of the ground-state functional $F[\gamma]$ of Eq. (5), the latter must also be symmetry dependent in this case.

This conclusion made for the prototype $\mathrm{H}_{4}$ molecule can be naturally generalized to the case of the dissociation of other symmetrical molecules, the ground state $\Psi^{I}$ of which belongs to a non-fully symmetrical irreducible representation $I \neq A_{1 g}$. In the course of the dissociation the potential energy curve $E^{I}(R)$ might well be crossed with that $E^{A_{1 g}}(R)$ of the fully-symmetrical state $\Psi^{A_{1 g}}$. If the $2 \mathrm{RDM}$ is modelled as a functional of occupation numbers, $\Gamma_{i j, k l}(n)$, then the ground-state functional $F[\gamma]$ becomes a piecewise sequence of the symmetry-dependent $F^{I}[\gamma]$. The present example shows clearly the spatial symmetry is important in this type of functional.

For the $\mathrm{H}_{2}+2 \mathrm{H}$ system, one can inverse Eq. (16) to obtain $c_{g}= \pm \sqrt{n_{1 \sigma}}$, along with $c_{u}$ from the normalization condition of the wave function. The exact functional $\Gamma_{i j, k l}(n)$ then can be established via the 2RDM Eqs. (19) and (20). The system can be regarded as a combination of two two-electron systems, i.e. two $\mathrm{H}_{2}$ molecules, while one of which is dissociated. 
The square-root $\mathrm{ON}$ dependence $\pm \sqrt{n_{1 \sigma}}$ is reminiscent of the paradigmatic exact two electron DMFT functional of Löwdin and Shull [15].

On the other hand, while the 2RDM Eqs. (21) and (22) provide the benchmark for $\Gamma_{i j, k l}(n)$, a simple inversion relation between the occupation numbers and $\mathrm{CI}$ coefficients cannot be established for the rhombus $\mathrm{H}_{4}$ system from Eq. (12) to Eq. (14), so an explicit functional $\Gamma_{i j, k l}(n)$ cannot be obtained via the 2RDM Eqs. (21) and (22). In their study of translationally invariant one-band lattice models, Schilling and Schilling [13] used the symmetry-adapted generalized Pauli constraints to establish possible inversions between the occupation numbers and CI coefficients. At the moment, we are not sure if one can follow the same line to obtain an explicit exact functional $\Gamma_{i j, k l}(n)$ for the rhombus $\mathrm{H}_{4}$ system. Even if it could be obtained, the precondition of symmetry-adapted generalized Pauli constraints would lead only to a symmetry-dependent functional. A universal DMFT functional which can be ap- plied to both the rhombus $\mathrm{H}_{4}$ system and the $\mathrm{H}_{2}+2 \mathrm{H}$ system is still to be discovered.

\section{SUMMARY}

From the present results it follows that the ground-state DMFT functional $F[\gamma]$ based on $\Gamma_{i j, k l}(n)$ retains its universality within a given symmetry. This is a significant limitation to the previous conception of model functional $F[\gamma]$ based on $\Gamma_{i j, k l}(n)$. If such a functional is used widely in simulations of state-crossing or structural (phase) transitions, caution should be exercised in checking its symmetry dependence.

\section{ACKNOWLEDGMENTS}

O. Gritsenko is supported by the National Science Centre of Poland under the Grant No. 2017/27/B/ST4/00756. J. Wang is supported by National Natural Science Foundation of China Grant No. 11274109.
[1] P. Hohenberg and W. Kohn, Phys. Rev. 136, B864 (1964).

[2] T. L. Gilbert, Phys. Rev. B 12, 2111 (1975).

[3] K. Burke, J. Chem. Phys. 136, 150901 (2012).

[4] S. Goedecker and C. J. Umrigar, Phys. Rev. Lett. 81, 866 (1998).

[5] M. Buijse and E. J. Baerends, Mol. Phys. 100, 401 (2002).

[6] O. V. Gritsenko, K. Pernal, and E. J. Baerends, J. Chem. Phys. 122, 204102 (2005).

[7] N. N. Lathiotakis and M. Q. L. Marques, J. Chem. Phys. 128, 184103 (2008).

[8] N. N. Lathiotakis, S. Sharma, J. K. Dewhurst, F. G. Eich, M. A. L. Marques, and E. K. U. Gross, Phys. Rev. A 79, 040501(R) (2009).

[9] M. Piris, Int. J. Quantum Chem. 106, 1093 (2006).

[10] M. Piris, J. M. Matxain, X. Lopez, and J. M. Ugalde, J. Chem. Phys. 132, 031103 (2010).

[11] M. Piris, Phys. Rev. Lett. 119, 063002 (2017).

[12] P. O. Löwdin and H. Shull, Phys. Rev. 101, 1730 (1956).
[13] C. Schilling and R. Schilling, Phys. Rev. Lett. 122, 013001 (2019).

[14] J. Wang and P. J. Knowles, Phys. Rev. A 92, 012520 (2015).

[15] M. Levy, Proc. Natl. Acad. Sci. USA 76, 6062 (1979).

[16] O. V. Gritsenko, Phys. Rev. A 97, 026501 (2018).

[17] J. Wang and P. J. Knowles, Phys. Rev. A 97, 026502 (2018).

[18] O. Gunnarsson and B. I. Lundqvist, Phys. Rev. B 13, 4274 (1976).

[19] U. von Barth, Phys. Rev. A 20, 1693 (1979).

[20] C. W. Wilson and W. A. Goddard, III, J. Chem. Phys. 51, 716 (1969).

[21] J. Paldus, P. Piecuch, L. Pylypow, and B. Jeziorski, Phys. Rev. A 47, 2738 (1993).

Correction: The column headings above the matrix in Eq. (21) were inadvertently offset during the final production cycle and have been fixed. 